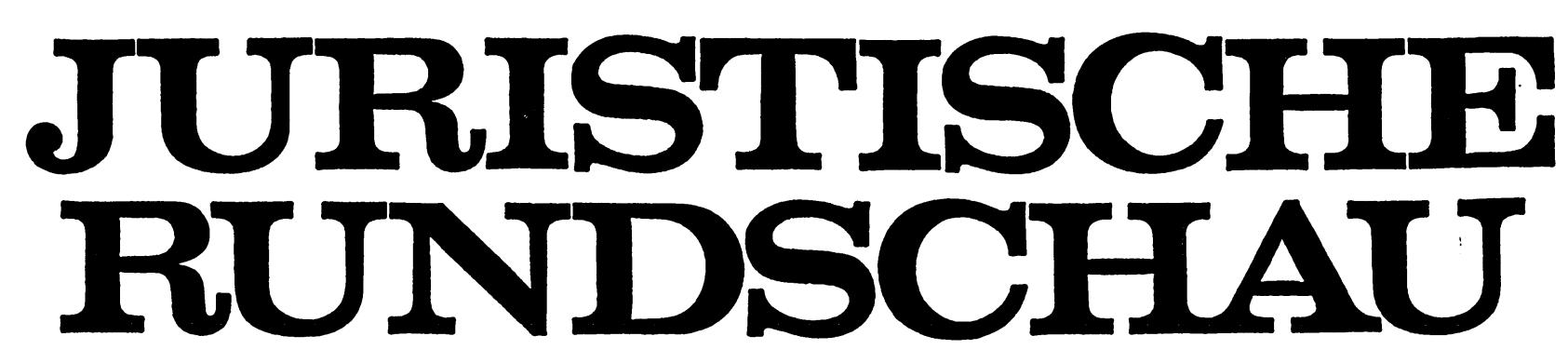

Schriftleitung: Rechtsanwalt Dr. Helwig Hassenpflug

\title{
Die Haftung beim Verkauf von Verträgen
}

\author{
Von Professor Dr. Ingo Koller, Passau
}

Der Beitrag befaßt sich mit Haftungsproblemen, die daraus resultieren, daß das BGB weder die Übertragbarkeit noch den Verkauf von Verträgen eigenständig geregelt hat und man daher auf komplizierte Analogieschlüsse und den Einsatz der Figur „Fehlen der Geschäftsgrundlage“ angewiesen ist. Behandelt werden drei Fallgruppen: Zum einen die Haftung des Verkäufers eines Vertrages, wenn der Dritte (Partner des verkauften Vertrages) seine Zustimmung zur Übertragung des Vertrages verweigert. Ferner die Haftung, falls der veräußerte Vertrag dem Käufer weniger Rechte verschafft, als er erwarten durfte. Schließlich wird eingehend die Fallgruppe untersucht, in der der Käufer nach Übernahme des Vertrages mit unerwarteten Verbindlichkeiten konfrontiert wird.

\section{Problem}

Das BGB schenkt der Übertragung von Verträgen nur als Annex zur Übertragung von Grundstücken $(\$ 571$ $\mathrm{BGB})$ und Betrieben sowie Betriebsteilen ( $\$ 613$ a BGB) Beachtung. Eine ältere Lehre schloß daraus, daß die Übertragung von Verträgen grundsätzlich auf dem Weg über eine Aufspaltung der Verträge im Wege der Zession der Rechte ( $\$ \int 398 \mathrm{ff}$. BGB) und Schuldübernahme der Pflichten ( $\$ \int 414 \mathrm{f}$. BGB) erfolgen müsse'. Der heute herrschenden Meinung zufolge ist diese Aufspaltung unnötig. Die Rechtsnachfolge in die Vertragsposition erfolgt durch Verfügungsvertrag zwischen Erwerber und Verkäufer über die gesamte Rechtsstellung des Veräußerers. In Analogie zu den $\int \mathbb{S} 414,415$ BGB bedarf dieser Verfügungsvertrag der Zustimmung des Gläubigers des veräußerten Vertrages ${ }^{2}$.

Dem Kausalgeschäft, das der Vertragsübernahme zugrunde liegt, hat man bislang - soweit ersichtlich keine besondere Aufmerksamkeit geschenkt ${ }^{3}$. Dies mag damit zusammenhängen, daß die Vertragsübernahme in aller Regel nicht isoliert, sondern im Zusammenhang mit anderen Geschäften wie dem Unternehmenskauf vereinbart wird. Gleichwohl lohnt es sich, der Haftung des Verkäufers eines isolierten Vertrages nachzugehen; denn allzu selten ist dieses Phänomen nicht. Man denke nur an entgeltliche Vereinbarungen des Mieters mit dem sog. Nachmieter oder an den Kauf von Bausparverträgen. Ferner kann die schuldrechtliche Dogmatik des isolierten Vertragskaufs wesentliche Einsichten für die Lösung von Problemen liefern, die bei der Veräußerung von Verträgen in komplexeren ${ }^{4}$ Zusammenhängen auftauchen.
Das BGB erfaßt mit dem Vertragstypus Kauf nur die Veräußerung von Sachen und von Rechten ( $\$ 433$ I BGB). Als kaufähnlich werden Verträge qualifiziert, die auf Veräußerung eines Gegenstandes gegen Entgelt gerichtet sind ( $\$ 445 \mathrm{BGB})$. Der herrschenden Meinung zufolge fallen darunter auch Vereinbarungen über die Veräußerung von Verträgen ${ }^{5}$, obwohl sich hier das Kausalgeschäft nicht allein darauf bezieht, dem Erwerber ein für ihn wertvolles Gut zu verschaffen ${ }^{6}$, sondern auch darauf, ihn mit Pflichten zu belasten, die dem Vertrag als einer Einheit entspringen. $\mathrm{Ob} \$ 445 \mathrm{BGB}$ der richtige Ansatzpunkt ist, kann indes letztlich auf sich beruhen. Auch wer sich auf $\$ 445$ BGB stützt, muß bei der ,entsprechenden Anwendung" der $\$ \$ S 433-444$ BGB berücksichtigen, daß der Käufer mit den Rechten zugleich Schulden „erwirbt“. Andererseits hat man auf die Wertungen des Kaufrechts selbst dann zurückzugreifen, wenn man der Ansicht ist, daß $\mathbb{4} 445 \mathrm{BGB}$ auf den hier diskutierten Vertragstypus nicht ganz paßt; denn der Kauf ist der sachnächste Vertragstyp ${ }^{7}$.

Vor diesem Hintergrund sind drei Fragenkreise eingehender zu untersuchen. Zum einen geht es um die Fallgruppe, in der die Veräußerung des Vertrages daran scheitert, daß der Gläubiger des zu veräußernden Vertrages seine Mitwirkung verweigert. Die zweite Fallgruppe ist dadurch gekennzeichnet, daß die dem Erwerber versprochenen Aktiva des Vertrages nicht existieren. Die meisten Schwierigkeiten bereitet schließlich diejenige

${ }^{1}$ BGH, NJW 1961, 453 (454).

2 BGH, NJW 1966, 499; WM 1973, 489; Münchener Kommentar (MünchKomm) - Roth, BGB (1979), § 398 Rdn. 4 m.w. Nachw.

${ }^{3}$ Rother, Festschrift Larenz (1973), S. 435 ff.; Grunewald, ZGR 1981, 622 (634ff.)

$\mathrm{Zu}$ denken ist hier in erster Linie an Unternehmenskäufe.

Münchener Kommentar (MünchKomm) - H. P. Westermann, BGB (1980), $\S 445$ Rdn. 4; Erman/Weitnauer, BGB (7. Aufl., 1981), § 445 Rdn. 1; Staudinger/ Köhler, BGB (1978), \433 Rdn.18, der dazu tendiert, den Vertragskauf als Rechtskauf zu behandeln.

6 Zum Begriff des Gegenstandes Staudinger/Dilcher, BGB (12. Aufl. 1979), vor $\$ 90$ Rdn. 3 ff.; Larenz, Allgemeiner Teil des deutschen Bürgerlichen Rechts, 5. Aufl., S. $250 \mathrm{f}$.

Ob im Fall von Leistungsstörungen beim Unternehmenskauf die spezifischen Wertungen des Kaufrechts wirklich so wenig Aussagekraft besitzen, wie Canaris (demnächst ZGR 1982 bei Fn. 88) behauptet, kann hier dahingestellt bleiben. Der Umstand allein, daß ein organisches Ganzes veräußert wird, zwingt nicht dazu, die einzelnen Elemente des Ganzen völlig in den Hintergrund treten zu lassen; denn die Informationsmöglichkeiten der Parteien beziehen sich immer auf die einzelnen Elementte und sind von der Struktur dieser Elemente abhängig (vgl. BGH, WM 1970,319 [320]). 
Fallgruppe, in der sich der Erwerber nach der Vertragsübernahme mit höheren Schulden (Passiva) konfrontiert sieht, als er erwartet hatte.

\section{Verweigerung der Zustimmung durch den Dritten}

Kommt die angestrebte Vertragübernahme nicht zustande, weil der Dritte ${ }^{8}$ die Zustimmung verweigert, so wäre es verfehlt, den Verkäufer des Vertrages immer nach den Regeln des anfänglichen Unvermögens haften zu lassen. Der Verkäufer eines Vertrages verspricht nicht uneingeschränkt den Erfolg „Übergang der Vertragsposition". Aus seiner "Erfolgsgarantie" ${ }^{\text {" } 9}$ sind vernünftigerweise diejenigen Teile seines Leistungsversprechens auszuklammern, deren Realisierung erkennbar nicht in seiner Herrschaftsmacht steht. Da die Vertragsübernahme partiell den Charakter einer Schuldübernahme besitzt und daher die Zustimmung eines Dritten erforderlich ist, muß der Käufer wissen, daß die Leistungsfähigkeit des Verkäufers von Faktoren in der Person des Dritten abhängig ist, die der Verkäufer nicht voll zu steuern oder auch nur hinreichend zuverlässig einzukalkulieren ver$\mathrm{mag}^{10}$. Die Verpflichtung des Verkäufers erstreckt sich daher - ähnlich den Konstellationen, in denen die Wirksamkeit des Vertrages bzw. des Erfüllungsgeschäftes von einer behördlichen Genehmigung abhängig is $t^{11}$ - lediglich auf eine angemessene Mitwirkung des Verkäufers bei der Besorgung der Zustimmung. Versagt der Dritte seine Zustimmung, ohne daß dem Verkäufer der Vorwurf einer schuldhaften Verletzung seiner Mitwirkungspflicht gemacht werden kann, so wird die Erfüllung des Vertrages insoweit unmöglich, ohne daß den Verkäufer eine Haftung trifft ${ }^{12}$. Etwas anderes gilt dort, wo der Verkäufer den Anschein erweckt, der Dritte habe seine Zustimmung bereits erteilt oder es liege in seiner Macht, den Dritten zur Zustimmung zu bewegen ${ }^{12 a}$.

Bei der Vertragsübernahme sind die Aktiva und Passiva des Vertrages regelmäßig untrennbar miteinander verknüpft. Es finden deshalb grundsätzlich die $\mathbb{\$} \$ 275,323$ I 1. HS BGB Anwendung. Nur in Fällen, in denen sich der Verkäufer ersichtlich mit einer Erfüllungsübernahme des Käufers begnügt hätte, können modifiziert die Regeln über die Teilunmöglichkeit eingreifen. \$323 I 2. HS BGB läßt sich nicht unmittelbar anwenden. Aus wirtschaftlicher Sicht stellt die Übernahme der dem Vertrag entspringenden Verbindlichkeiten durch den Käufer einen Teil der Gegenleistung des Käufers dar, auch wenn der Verkäufer „den Vertrag“ schlechthin schuldet. Kann der Käufer mangels Zustimmung des Dritten seine „Gegenleistung " in Form der sofortigen und endgültigen Entlastung von Verbindlichkeiten nicht erbringen, so ist entsprechend dem Rechtsgedanken des $\$ 323$ I 2. HS BGB die Vergütung in Geld heraufzusetzen. $\mathrm{Zu}$ diesem Zweck ist das Risiko, daß der Käufer seinen Pflichten aus der Erfüllungsübernahme nicht nachkommen wird, abzuschätzen. Da der Käufer auf diese Weise mit einer zusätzlichen Zahlungspflicht belastet wird, ist ihm seinerseits ein Rücktrittsrecht zuzubilligen.

\section{Haftung für den Bestand der vertraglichen Rechte}

Existiert der verkaufte Vertrag nicht, weil er z.B. wirksam angefochten worden ist oder weil er wegen Dissenses nicht wirksam zustandegekommen ist, so bestehen an einer Haftung des Verkäufers analog $\$ 437$ BGB keine Zweifel. Die Situation des Verkäufers und Käufers deckt sich mit der Situation des Käufers einer aus dem Vertrag abgeleiteten Forderung. Schwieriger sind diejenigen Fälle zu beurteilen, in denen der verkaufte Vertrag als solcher zwar Bestand hat, der Käufer aber dem Verkäufer erkennbar ${ }^{13}$ aus dem Vertrag weitergehendere Ansprüche zu erwerben erhofft hatte, als tatsächlich bestehen. Bevor hier auf eine Analogie zu $\ 437$ BGB zurückgegriffen wird, ist zuerst festzustellen, zu welcher Leistung sich der Verkäufer verpflichtet hat. Der Verkäufer verkauft einen bestimmten Vertrag. Geschuldet sind damit nicht unmittelbar all diejenigen Ansprüche, die dem Vertrag nach den Vorstellungen der Parteien entspringen sollen. Daraus folgt jedoch nicht, daß die Vorstellungen der Parteien über die Aktiva des Vertrages unerheblich wären. Vielmehr kennzeichnen sie den schutzwürdigen Erwartungshorizont des Käufers, der eine Gesamtheit von Aktiva und Passiva mit bestimmten rechtlichen Eigenschaften zu erwerben hofft. Fehlen bei Abschluß des Kaufvertrages einzelne der Aktiva, so ist $\$ 437$ BGB analog heranzuziehen; denn die ratio des $\$ 437$ BGB $^{14}$ ist auch hier erfüllt: Der Käufer muß sich in Hinblick auf den Bestand der mit dem verkauften Vertrag verbundenen Rechte weitgehend blindlings auf die Angaben des Verkäufers verlassen.

\section{Unerwartete Verbindlichkeiten}

\section{Diskussionsstand}

Die Frage, ob der Verkäufer eines Vertrages dafür haftet, daß sich der Käufer plötzlich mit unerwarteten Verbindlichkeiten aus dem von ihm übernommenen Vertrag konfrontiert sieht, ist bislang in dieser allgemeinen Form nicht eingehend diskutiert worden. Soweit ersichtlich hat bislang nur Grunewald ${ }^{15}$ diese Frage genauer geprüft. Sie kommt zu dem Ergebnis, daß der Verkäufer für das Auftauchen unerwarteter Verbindlichkeiten einzustehen hat, falls diese Verbindlichkeiten aus dessen früherem, auch objektivem Fehlverhalten herrühren. Intensiver ist die Auseinandersetzung mit dem Problem der Haftung für unerwartete Passiva im Rahmen der Diskussion um den Unternehmenskauf geführt worden. Hier wird zum Teil für eine Anwendung der Regeln über die Rechtsmängelhaftung plädiert, weil Verbindlichkeiten rechtliche Eigenschaften darstellten und wirtschaftlich die Existenz unerwarteter Passiva dem Verkauf nicht existierender Rechte ( $\$ 437$ BGB) gleichzustellen sei ${ }^{16}$. Dagegen wird eingewandt, daß unerwartete Verbindlichkeiten anders als typische Rechtsmängel die Nutzbarkeit der Aktiva nicht unmittelbar beeinträchtigten ${ }^{17}$ und daß die Rechtsmängelhaftung auf die Pflicht bezogen sei, das

${ }^{8}$ Die am Kausalgeschäft nicht beteiligte Partei des verkauften Vertrages.

9 Es wird hier davon ausgegangen, daß der Schuldner bei anfänglichem Leistungsunvermögen verschuldensunabhängig haftet. Koller, Die Risikozurechnung bei Vertragsstörungen in Austauschverträgen (1979), S. $265 \mathrm{ff}$. Vgl. ferner Köndgen, Selbstbindung ohne Vertrag (1981), S. 329. Zum Streitstand vgl. Staudinger/Löwisch, BGB (12. Aufl.), \306 Rdn. 30.

${ }_{10}$ Vgl. Koller, Risikozurechnung, a. a. O. (Fn. 9), S. 274

11 Staudinger/Löwisch, a. a. O. (Fn.9), 275 Rdn. $19 \mathrm{f}$; MünchKomm Roth, a.a. O. (Fn. 2), \$ 242 Rdn. 167 ff. jeweils m. w. Nachw.

${ }_{12}$ Anders für den Fall des Unternehmenskaufs zu generalisierend, BGH, WM 1970, 319 (320); 1975, 1166; Canaris, demnächst ZGR 1982 bei Fn. 75. Die Parallele zur Übertragung von isolierten Rechten paßt nicht, da Rechte grundsätzlich ohne Mitwirkung Dritter übertragbar, während Verträge grundsätzlich unübertragbar sind. Eine unmittelbare Anwendung des $\$ 437$ BGB scheidet ohnehin aus, da der Vertrag eine Einheit von Rechten und Verbindlichkeiten darstellt.

12a Insoweit zutreffend BGH, WM 1970, 319 (321).

13 Im Sinn einer verkehrserforderlichen Standards entsprechenden Aufmerksamkeit.

${ }^{14}$ Vgl. hierzu Koller, Risikozurechnung, a.a. O. (Fn.9), S. $104 \mathrm{ff}$

15 Grunewald, ZGR 1981, 622 (634 f.).

16 Loos, MDR 1962, 172 (173); H. Schlosser, JZ 1969, 337 (338); Flume, Eigenschaftsirrtum und Kauf (1949), S. $193 \mathrm{f}$

17 BGH, NJW 1980, 2408 (2409); Grunewald, ZGR 1981, 622 (624). 
Recht als solches zu verschaffen ${ }^{18}$. Vielmehr sei das Sachmangelgewährleistungsrecht analog anzuwenden. Der im Kaufvertrag vereinbarte Stand der Verbindlichkeiten sei nämlich als eine Eigenschaft des Unternehmens als einer Gesamtheit von Sachen, Rechten, immateriellen Werten und Passiva zu qualifizieren ${ }^{19}$, so daß unerwartete Verbindlichkeiten sowohl den Wert als auch die Gebrauchstauglichkeit beeinträchtigen könnten ${ }^{20}$. Canaris ${ }^{21}$ vertritt die Ansicht, daß die Regeln des Kaufrechts auf die sich beim Unternehmenskauf stellenden Probleme nicht zugeschnitten seien und daß daher generell auf die Geschäftsgrundlagenlehre zu rekurrieren sei.

Ohne daß hier auf die Besonderheiten des Unternehmenskaufs eingegangen werden muß, läßt sich für den Verkauf von Verträgen ebenfalls sagen, daß dort, wo unerwartet Forderungen gegen den Käufer $=$ Vertragsübernehmer erhoben werden, die spezifischen Regeln des Kaufrechts nicht passen. Die Vorschriften über die Rechtsmängelhaftung gehen in der Tat davon aus, daß der Verkäufer die Verschaffung von Aktiva in bestimmter rechtlicher Gestalt versprochen hat und daß er im Vergleich zu den $\$ \mathbb{S} 459 \mathrm{ff}$. BGB verschärft haften soll, weil sich der Käufer voll auf dessen Fähigkeiten, ihm diese Aktiva uneingeschränkt zu übertragen, verlassen muß. Im Hinblick auf die Verbindlichkeiten hat der Käufer indessen kein Interesse an deren Verschaffung. Vielmehr ist ausschließlich der Verkäufer daran interessiert, daß ihm diese Verbindlichkeiten abgenommen werden. Andererseits wird der Käufer des Vertrages durch unvorhergesehene Passiva auch nicht notwendig in der Ausnutzung der Vertragsaktiva behindert ${ }^{22}$. Gegen eine Parallele zur Sachmängelgewährleistung spricht wiederum, daß die im Vergleich zur Rechtsmängelhaftung geminderte ${ }^{23}$ Haftungsintensität des Verkäufers letztlich nur damit zu erklären ist, daß typischerweise auch der Käufer in der Lage ist, sich bei Vertragsschluß über die Existenz von Sachmängeln zu vergewissern ${ }^{24}$. Diese Informationschanchen stehen dem Käufer jedoch in Hinblick auf unbekannte Forderungen gerade nicht offen. $\mathrm{Da}$ er keine zuverlässigen Anhaltspunkte über den $\mathrm{Ab}$ schluß des verkauften Vertrages und die weitere in der Vergangenheit liegende Vertragsentwicklung besitzt, muß er sich so wie beim Verkauf eines Rechts weitgehend blindlings auf die Angaben des Verkäufers über den Stand der Passiva verlassen.

\section{Eigene Lösung}

Ausgangspunkt einer sachgerechten Lösung ist auch hier die Erkenntnis, daß der Käufer eines Vertrages, der faktisch Verbindlichkeiten des Verkäufers übernimmt, auf diese Weise eine Art Gegenleistung erbringt, die neben das im Kaufvertrag versprochene Entgelt tritt. Stellt sich später heraus, daß der Käufer größere Schulden als erwartet übernommen hatte, so muß der Käufer aus seiner Sicht für die Aktiva des Vertrages mehr als geplant aufwenden.

In einem ersten Schritt ist daher zu prüfen, in welchem Umfang sich der Käufer eines Vertrages zur Übernahme alter Verbindlichkeiten des Verkäufers verpflichtet hat. Die Regeln über den gesetzlichen Vertragsübergang geben hierfür wenig Aufschluß. So tritt einerseits der Käufer gemäß $\$ 571$ BGB nur in die Verbindlichkeiten ein, die nach dem Eigentumsübergang entstehen ${ }^{25}$. Andererseits haftet der Käufer $\ 613$ a BGB zufolge auch für Verbindlichkeiten, die bereits vor dem Betriebsübergang entstanden sind. Der Umfang der vom Käufer zu übernehmenden Verbindlichkeiten kann sich deshalb nur aus der Interpretation des konkreten Kaufvertrages ergeben. Wenn keine besonderen Anhaltspunkte existieren, wird man zunächst darauf abzustellen haben, daß der Vertrag als Einheit von Rechten und Pflichten verkauft worden ist. Hieraus folgt, daß der Käufer sich verpflichtet hat, die erkennbaren und die nichterkennbaren Verpflichtungen zu übernehmen. Dieser Deutung des Kausalgeschäftes entspricht das Verfügungsgeschäft. Der Dritte, der der Vertragsübernahme zustimmt, muß davon ausgehen können, daß der Käufer nach seiner Zustimmung zur Vertragsübernahme nicht nur für die ihm im Laufe der Vertragsverhandlungen erkennbar gewordenen Verbindlichkeiten, sondern für sämtliche, dem Vertrag entspringenden Forderungen haftet. Eine andere Frage ist es, ob der Verkäufer des Vertrages den Käufer von den unerwarteten Verbindlichkeiten freizustellen hat.

Das richtige Instrument zur Korrektur der Käuferverpflichtung ist die Lehre von der Geschäftsgrundlage. Dieses Instrument hat freilich den Nachteil, daß bislang nur wenig Konsens über Anwendungsbereich und Rechtsfolgen der Geschäftsgrundlagen-Figur erzielt worden ist. Es soll an dieser Stelle nicht versucht werden, die verschiedenen Ausprägungen der GeschäftsgrundlagenFigur aufzurollen ${ }^{26}$. Weitgehende Einigkeit besteht heute jedenfalls darin, daß die Belastung der Vertragsparteien mit dem Risiko eines Fehlens bzw. des Wegfalls der Geschäftsgrundlage wesentlich von Risikozurechnungserwägungen abhängt ${ }^{27}$. Auf einer etwas anderen Ebene liegt die Entscheidung über die Frage, ob man angesichts der Tatsache, daß sich die Parteien nun einmal vertraglich gebunden haben, die Rechtsfolgen nach Billigkeitsgrundsätzen bemißt oder im Interesse der Rechtssicherheit „entweder - oder" - Lösungen favorisiert. M.E. sollte das Schwergewicht wieder stärker auf die Rechtssicherheit gelegt werden, um den Justizapparat zu entlasten und die gerichtlichen Entscheidungen kalkulierbarer zu machen.

\section{a) Dem Käufer erkennbare Verbindlichkeiten}

Zunächst ist die Fallgruppe ins Auge zu fassen, in der die dem Vertrag entspringenden Verbindlichkeiten sowohl dem Verkäufer als auch dem Käufer zumindest dem(Mindest) Umfang nach erkennbar ${ }^{28}$ waren, der Käufer sie aber nicht erkannt hat. Für eine Haftung des Verkäufers spricht auf den ersten Blick, daß wohl der Verkäufer die besseren Informationen über Bestand und Höhe der vertragsimmanenten Schulden besessen hatte und daher für die Enttäuschung der Käufererwartungen einzustehen hat, es sei denn, daß dem Käufer analog

\footnotetext{
18 U. Huber, ZGR 1972, 395 (413 f.).

19 Wilhelm, Rechtsform und Haftung bei der juristischen Person (1981), S. 27 W. Müller, JuS 1975, 489 (491 f.); U. Huber, ZGR 1972, 395 (414); wohl auch Prölss, ZIP 1981, 337 (342f.).

20 H. P. Westermann, ZGR 1982, 45 (57)

21 Canaris, demnächst ZGR 1982 bei Fn. 90

${ }^{22}$ Etwas anderes gilt, wenn Verbindlichkeiten Zurückbehaltungsrechte auslö-

${ }^{23}$ Grundsätzlich nur Wandelung/Minderung. Zur Ausdehnung der Verkäuferhaftung mit Hilfe des Instruments der Zusicherung, Köndgen, Selbstbindung ohne Vertrag (1981), S. $331 \mathrm{ff}$

${ }^{24}$ Koller, Risikozurechnung, a.a.O. (Fn.9), S. 115 f. Vgl. auch Köndgen, Selbstbindung, a. a. O. (Fn. 23), S. 316. Ob dieses Prinzip im Weg der Rechtsfortbildung aufgegeben werden sollte, braucht hier nicht untersucht zu werden.

25 Staudinger/Emmerich, BGB (12. Aufl., 2. Bearb.), \$571 Rdn. 71.

26 Hierzu der Überblick von Roth in MünchKomm $\ 242 \mathrm{Rdn} .453 \mathrm{ff}$.

27 Vgl. BGH, NJW 1978, 2390; MünchKomm-Roth, a.a.O. (Fn. 2), \ 242 Rdn. 487 ff.; Staudinger/J. Schmidt, BGB (12. Aufl.), \242 Rdn. $833 \mathrm{ff}$.

28 Im Sinne verkehrserforderlicher Sorgfalt.
} sen. 
$\$ 460$ S. 2 BGB der Vorwurf grober Fahrlässigkeit gemacht werden kann. Diese Parallele zur Sachmängelhaftung ist jedoch nicht haltbar ${ }^{29}$. Der Verkäufer eines mit einem erkennbaren Sachmangel behafteten Kaufobjektes unterliegt der Gewährleistung, weil er typischerweise über einen Informationsvorsprung verfügt $t^{30}$, also erst recht die Fehlerhaftigkeit des Kaufobjekts erkennen konnte $^{31}$, und nach der subjektiven Fehlerlehre eine Sache mit bestimmten Solleigenschaften verkauft hat ${ }^{32}$. Gleiches gilt nach einer mehr objektiven Fehlertheorie ${ }^{33}$, falls dem Verkäufer die Erwartung des Käufers, eine Sache mit bestimmten Eigenschaften zu erhalten, erkennbar $w^{34}{ }^{34}$, und er nicht deutlich gemacht hatte, daß er insoweit keinen Beitrag zur Realisierung der Käufererwartungen leisten wolle ${ }^{35}$. Anders ist die Situation beim Verkauf von Verträgen. Hier kann der Verkäufer davon ausgehen, daß der Käufer die in ihrem Umfang ${ }^{36}$ erkennbaren Vertragsverbindlichkeiten auch übernehmen will. Er hat daher keinerlei Anlaß, seinen Informationsvorsprung bezüglich der Art und des Ausmaßes der dem Vertrag entspringenden Verbindlichkeiten ins Spiel zu bringen, um die ihm erkennbare Planung des Käufers zu realisieren ${ }^{37}$. Eine Haftung des Verkäufers ist daher erst dort am Platz, wo es aus der Sicht des Verkäufers evident ist, daß der Käufer objektiv erkennbare Vertragsverbindlichkeiten übersehen hat.

\section{b) Dem Käufer unerkennbare Verbindlichkeiten}

Brisanter sind diejenigen Fälle, in denen für den Käufer - wie häufig - die dem Vertrag entspringenden Verbindlichkeiten nicht vollständig erkennbar sind. Hier ist zwischen zwei im Kern unterschiedlichen Konstellationen zu differenzieren.

In der ersten Fallgruppe macht der Verkäufer keinen Versuch, den Käufer darüber aufzuklären ${ }^{38}$, daß seine Unterlagen unvollständig sein könnten oder daß noch zusätzliche Verbindlichkeiten begründende Umstände auftauchen können ${ }^{39}$. Hier wird sich der Käufer regelmäBig - so wie der Käufer einer Sache auf die Mangelfreiheit des Kaufobjektes - darauf verlassen, daß ihm die relevanten Verbindlichkeiten umfassend bekannt geworden sind. Die Tatsache, daß bei jeder Vertragsübernahme mit unerkennbaren Verbindlichkeiten zu rechnen ist, steht dem nicht entgegen; denn der Käufer einer Sache muß ja ebenfalls aufgrund allgemeiner Erfahrung immer mit einem Mangel rechnen, ohne daß dies die Haftung des Verkäufers berührt.

In der zweiten Fallgruppe macht der Verkäufer deutlich, daß seine Informationen über Umfang der Verbindlichkeiten nicht umfassend sind, oder die Parteien gehen im Rahmen der Kaufverhandlungen ersichtlich davon aus, daß mit zusätzlichen Forderungen zu rechnen ist ${ }^{40}$, deren Höhe allerdings nicht exakt quantifiziert werden kann. Im folgenden soll zunächst die Haftung des Verkäufers in der ersten Fallgruppe untersucht werden.

\section{aa) Das Risiko unbekannter Verbindlichkeiten war nicht Gegenstand der Vertragsverhandlungen}

Die Tatsache allein, daß dem Käufer des Vertrages die Existenz bestimmter zusätzlicher Verbindlichkeiten nicht erkennbar wurde ${ }^{41}$ und dieses Risiko auch nicht in die Vertragsverhandlungen einging, sondern daß der Käufer ersichtlich auf der Basis verhandelte, daß mit der Existenz weiterer Verbindlichkeiten nicht zu rechnen sei, rechtfertigt es nicht, den Verkäufer mit der Haftung zu belasten. Für die Haftung des Verkäufers ist nämlich auch ein Risikozurechnungsgrund erforderlich ${ }^{42}$.

Als Risikozurechnungsgrund kommt in erster Linie ein Verschulden des Verkäufers in Betracht. Dort, wo dem Verkäufer der Vorwurf gemacht werden kann, daß ihm als Partei des zu veräußernden Vertrages das Ausmaß der vom Käufer übernommenen Verbindlichkeiten hätte bekannt sein müssen, ist eine Haftung des Verkäufers regelmäßig ${ }^{43}$ unproblematisch. $\mathrm{Zu}$ ihr gelangt man schon auf der Grundlage der Regeln über die c.i.c.; denn es gehört zu den vorvertraglichen Pflichten des Verkäufers, den Käufer sorgfältig über die dem Vertrag entspringenden Forderungen aufzuklären, da sich der Käufer anderweit nur schwer und unzureichend Gewißheit über die mit der Vertragsübernahme verbundenen Belastungen zu verschaffen imstande ist.

Nicht immer kann aber dem Verkäufer der Vorwurf einer Verletzung seiner Informationspflichten gemacht werden. Es sind auch Konstellationen vorstellbar, in denen später Forderungen auftauchen, die auch dem Verkäufer bei Abschluß des Kaufvertrages unerkennbar waren. Man denke etwa an Schadensersatzansprüche des Vermieters, die aus einer vertragswidrigen Benutzung des Mietobjektes durch den Verkäufer des Mietvertrages herrühren und erst nach Übernahme des Vertrages durch den Käufer zu erkennbaren Schäden geführt haben. In diese Kategorie gehören auch Fälle, in denen der Käufer wider Erwarten aus einer vom Verkäufer des Vertrages abgegebenen Garantie in Anspruch genommen wird, Verbindlichkeiten durch den Zugang von Willenserklärungen begründet worden sind, die dem Verkäufer nicht zur Kenntnis gelangt sind oder durch Hoheitsakt mit rückwirkender Kraft vertragliche Ansprüche begründet werden ${ }^{44}$. Diese Fälle lassen sich zum Großteil mit Hilfe der $\$ 437$ BGB zugrundeliegenden Wertung ${ }^{45}$ lösen. $\$ 437$ BGB ordnet eine verschuldensunabhängige Haftung des Verkäufers für den Bestand des verkauften Rechts an, weil sich der Käufer eines Rechts typischerweise nicht hinreichend zuverlässig über die Existenz des Rechts zu vergewissern vermag, da er dazu die Interna des Verkäufers und sämtliche in der Vergangenheit liegende Faktoren aufrollen müßte, die für den Bestand des

29 Unzutreffend im Ansatz daher Grunewald, ZGR 1981, 622 (634ff.).

30 Zur ratio der $\$ \$ 459 \mathrm{ff}$. BGB Koller, Risikozurechnung, a. a. O. (Fn.9) S. 115 ff., 142 ff.; Prölss, ZIP 1981, 337 (339 ff.).

31 Vgl. Koller, Risikozurechnung, a.a.O. (Fn. 9), S. 149 Fn. 239

32 Grundlegend Flume, Eigenschaftsirrtum, a. a. O. (Fn. 16), S. 109 f.

33 Larenz, Schuldrecht II (12. Aufl. 1981) \$ 41 II e m. Nachw.; Canaris, demnächst ZGR 1982 bei Fn. 7. In der Tendenz auch Koller, Risikozurechnung a. a. O. (Fn. 9), S. $146 \mathrm{ff}$

${ }^{34}$ Koller, Risikozurechnung, a.a. O. (Fn. 9), S. 149 f.

35 Vgl. Flume, Eigenschaftsirrtum, a. a. O. (Fn. 16), S. 164 f.; Koller, Risikozurechnung, a.a. O. (Fn. 9), S. 151.

${ }^{36}$ Hierfür genügt es, daß z. B. aller Erfahrung nach mit Sicherheit in gewisser Höhe zusätzliche Verbindlichkeiten zu erwarten sind. Diese leicht quantifizierbaren Verbindlichkeiten hat der Käufer zu berücksichtigen. Insoweit zutreffend Grunewald, ZGR 1981, 622 (636f.).

37 Anders im Ergebnis wohl Staudinger/J. Schmidt, a.a.O. (Fn. 27), $\$ 242$ Rdn. 1006.

${ }^{38}$ Diese Aufklärung ist natürlich nicht erforderlich, falls der Käufer davon ausgehen muß, daß in einer bestimmten Marge unbekannte Verbindlichkeiten auftauchen werden. Diese Verbindlichkeiten gehören zum Kreis der erkennbaren Forderungen.

${ }^{39}$ Hier ist z.B. daran zu denken, daß der Verkäufer des Vertrages damit rechnen muß, aus einer Garantie in Anspruch genommen zu werden.

40 Näher dazu unten bei Fn. 56.

41 Falls sie erkennbar werden mußte, gilt das bei Fn. 36 Gesagte.

42 Freiwillige Selbstbindung im Sinne der Untersuchung Köndgens, Selbstbindung, a.a.O. (Fn. 23), S.334 ff. genügt hierfür nicht. Für eine Haftung sind zusätzliche Zurechnungselemente erforderlich, da es um das Risiko geht, daß die geweckten Erwartungen nicht erfüllt werden können und ein Schaden ersetzt werden muß.

${ }^{43}$ Zur Ausnahme bei Fn. 56.

44 Z.B. Ansprüche auf Erstattung höherer Mehrwertsteuern.

45. Vgl: dazu Koller, Risikozurechnung, a. a. O. (Fn. 9), S. 104 ff. 
Rechts erheblich sein könnten. Auf der anderen Seite liegen die für den Bestand des Rechts wesentlichen Umstände zum größten Teil in der Sphäre des Verkäufers oder haben sich in der Vergangenheit dort zuerst ausgewirkt. Der Verkäufer ist daher im Vergleich zum Käufer des Rechts als einziger in der Lage, die Gefahr zu steuern, daß das verkaufte Recht wider Erwarten nicht existiert ${ }^{46}$. Die gleiche Verteilung der Informations- und Beherrschungsmöglichkeiten ist bei der Übernahme von Verbindlichkeiten im Rahmen eines Vertragsüberganges zu beobachten. Auch hier besitzt der Käufer regelmäßig keinerlei Chance, das Ausmaß der übernommenen Verbindlichkeiten zuverlässig zu eruieren. Auf der anderen Seite liegen die Umstände, aus denen sich die unerwarteten Vertragsverbindlichkeiten ergeben, typischerweise in der Sphäre des Vertragsverkäufers oder haben sich dort bemerkbar gemacht. Das gilt z. B. für die vertragswidrige Benutzung des Mietobjekts, den Zugang von Willenserklärungen ebenso wie für die eine Garantiehaftung auslösenden Faktoren einer Schlechtleistung ${ }^{47}$ oder Faktoren, die für die Auslegung des verkauften Vertrages maßgeblich sind. Man wird einwenden, daß der nachträgliche Eintritt des Schadens beim Partner des verkauften Vertrages außerhalb der Sphäre des Verkäufers angesiedelt sei. Dies ist zwar richtig, doch ist bei der Risikoverteilung zu berücksichtigen, daß der Verkäufer immerhin als einziger in der Lage ist, das Risiko abzuschätzen und einzukalkulieren, daß sich später ein Schaden ereignen wird ${ }^{48}$. Diese Risikokalkulation wird sich freilich nur selten auf handfeste statistische Daten stützen können. Gerade die von festen Erfahrungswerten losgelöste Risikoeinschätzung ist jedoch Ausdruck eines besonderen Geschicks ${ }^{49}$, das bei der Zuordnung von Risiken erwartet wird. So wird niemand dem Gattungschuldner entgegen $\$ 279$ BGB die Berufung darauf gestatten, daß dic plötzlichen Preissteigerungen auf den Beschaffungsmärkten über dem statistischen Durchschnitt der Preissteigerungen der letzten Jahre liegen. Zum anderen ist zu berücksichtigen, daß der Käufer des Vertrages in derartigen Konstellationen normalerweise über keinerlei Anhaltspunkte verfügt, anhand derer er sich über das Ausmaß des übernommenen Risikos klar werden könnte. Von daher erscheint es als sachgerecht, den Verkäufer eines Vertrages grundsätzlich auch für die Nichtexistenz von Verbindlichkeiten, die bei Abschluß des Kaufvertrages nach Art und/oder Umfang unerkennbar waren, haften zu lassen. Die Haftung geht in Parallele zu $\$ 437$ BGB auf das positive Interesse des Käufers, d. h. Befreiung von den unerkennbaren Verbindlichkeiten.

Die Zurechnung aufgrund der dem Verkäufer des Vertrages offenstehenden Informationsmöglichkeiten endet dort, wo nach Vertragsschluß gänzlich unvorhersehbare $^{50}$ Forderungen auftauchen. Auch in derartigen Fällen wird man grundsätzlich aber den Verkäufer verpflichten müssen, den Käufer zumindest partiell von diesen Verbindlichkeiten zu befreien. Aus der Sicht des Käufers eines Vertrages, der sich plötzlich mit unvorhersehbaren Verbindlichkeiten konfrontiert sieht, stellt dies eine gravierende Äquivalenzstörung dar; denn der Käufer muß - wirtschaftlich gesehen - mehr Aufwand zur Erfüllung seines Versprechens treiben, als er bei Abschluß des Kaufvertrages geplant hatte. Er befindet sich der Sache nach in der gleichen Lage, wie der Verkäufer einer Ware, der sich aufgrund unvorhersehbarer Umstände $\mathrm{zu}$ wesentlich höheren Preisen auf dem Markt eindecken muß. In diesen Fällen ist es anerkannt, daß sich der Verkäufer auf den Wegfall der Geschäftsgrundlage berufen kann. Die herrschende Meinung gestattet dies allerdings erst dann, wenn die Äquivalenzstörung besonderes Gewicht besitzt, d.h. einen bestimmten Schwellenwert überstiegen hat, der mit Werten zwischen $12 \%$ und mehr als $70 \%$ der Erhöhung des geplanten Aufwandes beziffert wird ${ }^{51}$ s2.

Der Rechtssicherheit wäre gedient, wenn man diesen Schwellenwert möglichst niedrig ansetzt, da sonst rohe Quantifizierungen ohne innere Überzeugungskraft nicht $\mathrm{zu}$ vermeiden sind ${ }^{53}$. Es empfiehlt sich hier, eine Parallele zu $\int 459$ I 2 BGB zu ziehen und dem Käufer zu erlauben, sich auf die Belastung durch unvorhersehbare Verbindlichkeiten zu berufen, wenn die Zusatzbelastung einigermaßen ins Gewicht fällt. Wenig Klarheit herrscht auch in bezug auf die konkreten Rechtsfolgen in der hier zur Diskussion stehenden Unterfallgruppe. Die herrschende Meinung plädiert dafür, die Rechtsfolgen flexibel nach Billigkeitsgrundsätzen zu bestimmen und nach Möglichkeit die Zusatzbelastung zwischen den Parteien aufzuteilen $^{54}$. Die Überzeugungskraft dieser Form der Rechtsfolgenbestimmung hängt davon $a b$, wie hoch man die Rechtssicherheit bewertet und welche Bedeutung man dem Umstand beilegt, daß der Käufer im Interesse des Verkäufers ein Risiko auf sich genommen hat, das der Verkäufer des Vertrages sonst hätte selbst tragen müs$\operatorname{sen}^{5 s}$.

\section{bb) Das Risiko unbekannter Verbindlichkeiten war Ge- genstand der Vertragsverhandlungen}

Eröffnet der Verkäufer dem Käufer im Rahmen der Vertragsverhandlungen, daß mit zusätzlichen Verbindlichkeiten gerechnet werden müsse, oder gehen beide Parteien aufgrund allgemeiner Erfahrung oder besonderer Umstände davon aus, daß der den Vertragsverhandlungen zugrunde gelegte Stand der Verbindlichkeiten nicht exakt sei, so hängt die Haftung des Verkäufers

${ }^{46}$ Im Sinn abstrakter Beherrschbarkeit. Bei dieser Form der Beherrschbarkeit geht es anders als bei Sorgfaltspflichtsverletzungen nicht darum, daß der Schuldner die Gefahr mit Hilfe eines zumutbaren, im Einzelfall konkret zu bestimmenden Aufwandes hätte bannen können. Die verschuldensunabhängige Zurechnung aufgrund abstrakter Beherrschbarkeit basiert auf der Überlegung, daß der Schuldner typischerweise über Informationen verfügt oder den Zugang zu Informationen hat, die es ihm ermöglichen, ohne normative Vorgaben (verkehrserforderliche Standards) die optimale Schadensabwehr zu bestimmen. Ausgangspunkt ist die Belastung des Schuldners mit jedem Schaden ohne Rücksicht auf das Maß des zur Gefahrenabwehr erforderlichen Aufwandes. Ist der potentielle Schaden höher als der zur Verhinderung des Schadens nötige Aufwand, so wird der Schuldner sich aus simplen Rentabilitätsüberlegungen für eine Gefahrenabwehr entscheiden. Dort, wo der potentielle Schaden die Gefahrenabwehr nicht lohnt, wird der Schuldner die der potentielle Schaden die Gefahrenabwehr nicht lohnt, wird der Schuldner die erreicht, daß der Schuldner überall dort, wo eine Gefahrenabwehr rentabel ist, sich bemüht, drohende Gefahren über den Standard der Sorgfaltspflichten hinaus auszuschalten (eingehend zum Prinzip abstrakter Beherrschbarkeit Koller, Risikozurechnung, a.a.O. (Fn.9), S. 78 ff.).

$47 \mathrm{Vgl}$. Grunewald, ZGR 1981, 635.

48 Es geht hier um das Absorptionsprinzip, demzufolge derjenige Teil das Risiko auf sich zu nehmen hat, der das Risiko besser einzukalkulieren und gegebenenfalls zu streuen vermag. Eingehend dazu Koller, Risikozurechnung, a. a. O. (Fn. 9), S.89 ff

49 Vgl. Philipp, Risiko und Risikopolitik (1967), S. 31; Koller, Risikozurechnung, a.a. O. (Fn. 9), S. $221 \mathrm{f}$.

so Zur Bedeutung des Begriffs Vorhersehbarkeit Koller, Risikozurechnung a. a. O. (Fn. 9), S.217 ff. In dem hier zur Diskussion stehenden Zusammenhang geht es eigentlich um Fragen der Erkennbarkeit. Aus der Planungsperspektive der Parteien im Moment des Vertragsschlusses kommt es auch insoweit auf Wahrscheinlichkeitsüberlegungen an.

51 Vgl. BGH, NJW 1976, 142 f.; WM 1969, 1021; KG, NJW 1958, 910; Kegel, Verhandlungen des Deutschen Juristentages (1953), Bd. I, S.202; Wieacker, Festschrift Wilburg I (1965), S.251; MünchKomm-Roth, a.a.O. (Fn. 2), $\$ 242$ Rdn. $535 \mathrm{ff}$.

52 Canaris, demnächst ZGR 1982 bei Fn. 29 beziffert den Schwellenwert mit ca. $15 \%$.

53 Vgl. Koller, Risikozurechnung, a. a. O. (Fn. 9), S. 229.

54 Vgl. MünchKomm-Roth, a. a. O. (Fn. 2), \242 Rdn. 494 ff. m. Nachw.

55 Vgl. Koller, Risikozurechnung, a.a. O. (Fn. 9), S. $241 \mathrm{f}$. 
davon $a b$, wie sich die Parteien auf diese Situation der Ungewißheit eingelassen haben.

Zunächst ist an die Konstellationen zu denken, in denen der Verkäufer wegen der unüberschaubaren Lage Preiszugeständnisse macht. Der Sache nach heißt dies, daß das Risiko unbekannter Verbindlichkeiten in Form eines Risikoabschlags ${ }^{56}$ quantifiziert wird. Der Verkäufer gibt mit anderen Worten zu erkennen, daß er die Übernahme des Risikos durch den Käufer mit einem Preisabschlag honorieren wolle. Auf der anderen Seite ist das Verhalten des Käufers, der sich darauf einläßt, nur dahin zu deuten, daß er den Abschlag für eine ausreichende Wagnisprämie hält $t^{57}$. Werden später gegen den Käufer aus dem Vertrag unerwartete Verbindlichkeiten geltend gemacht, so kann er sich nicht darauf berufen, daß er das Risiko zu niedrig eingeschätzt habe. Es wäre seine Sache gewesen, im Rahmen der Vertragsverhandlungen einen höheren Abschlag durchzusetzen oder wenn er glaubte, den Abschlag wegen unzureichender Informationen nicht zuverlässig genug kalkulieren zu können, auf einer Haftung des Verkäufers zu bestehen ${ }^{58}$. Eine Ausnahme gilt nur für gänzlich unvorhersehbare Verbindlichkeiten. Das Risiko derartiger Verbindlichkeiten ist mit Sicherheit in den Risikoabschlag nicht eingegangen, so daß es nach allgemeinen Grundsätzen ${ }^{59}$ dem Verkäufer zur Last fällt.

Die Verhandlungen können aber auch derart geführt werden, daß beide Parteien gemeinsam abzuschätzen versuchen, mit welchen zusätzlichen Verbindlichkeiten zu rechnen ist und auf dieser Basis die Preisverhandlungen führen. Hier gründen beide Parteien ihre Äquivalenzentscheidung bewußt auf die Annahme, daß eine bestimmte Entwicklung am wahrscheinlichsten sei und tragen der Tatsache, daß immer auch mit abweichenden Entwicklungen zu rechnen sei, bewußt keinerlei Rechnung. In derartigen Konstellationen hat der $\mathrm{BGH}^{60} \mathrm{zu}$ Recht in großzügiger Weise den Wegfall der Geschäftsgrundlage bejaht; denn die Parteien haben hier zu erkennen gegeben, daß das Risiko unbekannter Verbindlichkeiten nicht abschließend gelöst sein soll, weil weder der Käufer noch der Verkäufer bereit war, ein besonderes Wagnis einzugehen ${ }^{61}$. Die Konsequenz ist, daß dort, wo die tatsächliche Entwicklung erheblich von den dem Vertragsschluß zugrunde gelegten Annahmen abweicht, entweder der Verkäufer den Käufer von zusätzlichen Verbindlichkeiten zu befreien oder umgekehrt der Käufer dem Verkäufer einen höheren Kaufpreis zu zahlen hat, falls die wahren Verbindlichkeiten hinter den erwarteten zurückbleiben. Diese Fallgruppe wird freilich recht selten auftreten; denn in aller Regel wollen die Parteien, die sich bestimmter Risiken bewußt geworden sind und darüber verhandelt haben, abschließende Regelungen. Man wird also immer eingehend zu prüfen haben, ob der Verkäufer nicht verdeckte Preiszugeständnisse gemacht hat, die z.B. in der Form erfolgen können, daß bei den Preisverhandlungen auch verhältnismäßig unwahrscheinliche Verbindlichkeiten voll in Anschlag gebracht werden oder die Aktiva des Vertrages niedriger bewertet werden.

Schließlich ist die Fallgruppe zu betrachten, in der der Verkäufer zu erkennen gibt, daß er für die Vollständigkeit der Information über die offenen Verbindlichkeiten nicht einzustehen gedenke. Dies muß nicht ausdrücklich erfolgen, sondern kann auch konkludent etwa in der Form erfolgen, daß der Verkäufer auf die Unvollständigkeit seiner Unterlagen oder darauf hinweist, es müsse mit dem unerwarteten Auftauchen weiterer Forderungen gerechnet werden. Hier ist es Sache des Käufers, sein Interesse durch hinreichende Risikoabschläge zu sichern oder falls er glaubt, daß er zu wenig Anhaltspunkte für eine Berechnung dieser Abschläge besitzt, auf eine Haftungsübernahme seitens des Verkäufers zu drängen. Läßt sich der Käufer darauf ein, den Vertrag ohne eine Haftungsübernahme durch den Verkäufer zu erwerben, so hat er das Risiko zusätzlicher Verbindlichkeiten zu tragen, soweit es vorhersehbar war. Unvorhersehbare Verbindlichkeiten gehen hingegen ${ }^{62}$ normalerweise nach allgemeinen Regeln ${ }^{63}$ zu Lasten des Verkäufers; denn man wird die Erklärung des Verkäufers eng dahin interpretieren müssen, daß er aufgrund besonderer Umstände lediglich nicht bereit sei, das Risiko einer Unzulänglichkeit seines Geschäftskreises auf sich zu nehmen.

\section{Zusammenfassung}

1. Der Verkauf von Verträgen als Einheit von Forderungen und Verbindlichkeiten ist im BGB nicht adaequat erfaßt. Die Haftung des Verkäufers erschließt sich daher nur im Wege von Analogieschlüssen und unter Anwendung der Geschäftsgrundlagen-Figur.

2. Der Verkäufer eines Vertrages haftet grundsätzlich nicht dafür, daß der Dritte (Partner des veräußerten Vertrages) seine Zustimmung zur Übertragung des Vertrages erteilt.

3. Der Verkäufer haftet analog $\ 437$ BGB dafür, daß der Käufer mit dem Vertrag die erwarteten Forderungen erwirbt.

4. Die Haftung des Verkäufers für unerwartete Verbindlichkeiten aus dem verkauften Vertrag richtet sich primär nach den Verhandlungen der Parteien.

a) Wurde die Existenz unbekannter Verbindlichkeiten zum Gegenstand der Vertragsverhandlungen gemacht, so hängt die Haftung des Verkäufers - gänzlich unvorhersehbare Verbindlichkeiten ausgeklammert - davon ab, daß der Verkäufer sich auf keine Preiszugeständnisse einläßt und/oder nicht deutlich macht, daß er für unbekannte Verbindlichkeiten auch nicht einzugestehen gedenke. Die Regeln über das Fehlen der Geschäftsgrundlage sind lediglich dort anzuwenden, wo die Parteien der Gefahr unbekannter Verbindlichkeiten bewußt keine endgültige Rechnung getragen haben.

b) Wurde die Gefahr unbekannter Verbindlichkeiten nicht zum Gegenstand der Vertragsverhandlungen gemacht, so haftet der Verkäufer analog $\$ 437$ BGB auf das positive Interesse, wenn für ihn, nicht aber für den Käufer, die Existenz der Verbindlichkeit bei verkehrserforderlicher Sorgfalt erkennbar oder deren Existenz vorhersehbar war. Dort, wo nachträglich gänzlich unvorhersehbare Verbindlichkeiten auftauchen, ist das Risiko nach den Regeln der Geschäftsgrundlage m.E. in der Weise auf den Verkäufer abzuwälzen, daß der Käufer gegen den Verkäufer einen Befreiungsanspruch erwirbt.

\footnotetext{
56 Der Risikoabschlag muß das gesamte Risiko zusätzlicher Verbindlichkeiten, nicht nur das Risiko widerspiegeln, daß der Käufer auf den Freistellungsanspruch gegen den Verkäufer angewiesen ist.

57 Vgl. BGH, WM 1969, 527; WM 68, 1010 (1011).

58 Vgl. BGH, WM 1969, 527; MünchKomm-Roth, a.a.O. (Fn. 2), \242 Rdn. 569 .

59 Dazu oben bei Fn. 50

60 BGH, WM 1968, 1010 (1011); vgl. auch MünchKomm-Roth, a.a.O. (Fn. 2), \ 242 Rdn. 568.

61 MünchKomm-Roth, a. a. O. (Fn. 2), $\$ 242$ Rdn. 570, 612; Koller, Risikozurechnung, a.a.O. (Fn. 9), S. $380 \mathrm{f}$.

62 Vom Ansatz her zutreffend Staudinger/J. Schmidt, a. a. O. (Fn. 27), $\$ 242$ Rdn. 892.

63 Dazu oben bei Fn. 50
} 